\title{
Midiendo los usos y recursos tecnológicos de las Administraciones locales*
}

\author{
J. Ignacio Criado \\ Investigador \\ Departamento de Ciencias Políticas y de la Administración II \\ Universidad Complutense de Madrid \\ Contactar en: www.jicriado.com.
}

Sumario: 1. INTRODUCCIÓN. 2. LAS TECNOLOGÍAS EN LAS ADMINISTRACIONES LOCALES. 3. UN ANÁLISIS DE LOS AYUNTAMIENTOS DE LA COMUNIDAD DE MADRID. Diseño de la investigación. Algunos datos de partida. Interacciones entre gestión pública local y e-Administración. Motivaciones para la creación de páginas web y beneficios de su desarrollo. Barreras contra la e-Administración local. 4. REFLEXIONES SOBRE EL CUESTIONARIO Y LA e-ADMINISTRACIÓN LOCAL. 5. CONCLUSIÓN. BIBLIOGRAFÍA.

\section{INTRODUCCIÓN}

Las Administraciones Públicas han adoptado tecnologías a través de proyectos diversos y generalmente muy costosos, sin embargo, el alcance de los mismos no se ha solido contrastar, especialmente, en el ámbito local. Muchas de las iniciativas de e-Administración se inscriben dentro del sector público local, de manera que las tecnologías se han tendido a estudiar de forma habitual en las Administraciones locales gracias a las posibilidades ofrecidas por el potencialmente alto número de unidades de análisis. Dentro de esos estudios, una de las técnicas de recogida de datos más utilizadas ha sido el cuestionario, sobre todo, cuando se buscaban conclusiones generalizables sobre un número limitado de dimensiones y variables de las organizaciones locales teniendo en cuenta la perspectiva de los usuarios internos. En este artículo se pone en evidencia la importancia de este tipo de estudios para conocer la realidad de las tecnologías en las Administraciones locales y se plantea como ejemplo una investigación desarrollada por el autor dentro de los municipios de una región española.

Uno de los factores que más se ha utilizado para justificar los cambios en el seno de la gestión de las Administraciones locales en los últimos años es la adopción y difusión de las Tecnologías de la Información y la

\footnotetext{
* Este trabajo ha sido posible gracias a una beca de Formación de Personal Investigador de la Comunidad de Madrid, co-financiada por el Fondo Social Europeo, organismos a los que el autor agredece su apoyo.
} 
Comunicación (TIC). Hace décadas, la adopción por las Administraciones locales de las últimas aplicaciones tecnológicas se justificaba atendiendo a su capacidad para incrementar la productividad basada en una reducción de los efectivos humanos y en un aumento del trabajo realizado (BELLAMY y TAYLOR, 1998; HeEks, 1999; MargetTS, 1999). Recientemente, los fines perseguidos por las Administraciones locales se orientan hacia el ciudadano y la mejora de la calidad de los servicios públicos (ARENILLA, 2003; Dunleavy y MargetTs, 2002; Ramió, 2002). Dentro de esa dinámica de cambio, una de las preocupaciones de los autores que analizan la e-Administración local consiste en establecer patrones comunes de medida que permitan evaluar esa realidad en contextos diversos y utilizando diseños de investigación asimilables a diferentes tradiciones administrativas (CASTElls y OlLÉ, 2004; CRIADO y RAMILO, 2003a; SALVADOR, 2004). Las siguientes páginas pretenden contribuir en esa tarea a través de una aproximación a la contribución específica del cuestionario como instrumento de medida dentro de los estudios sobre las tecnologías en las Administraciones locales.

El artículo opera ofreciendo como marco de referencia un repaso por algunas investigaciones que han utilizado el cuestionario como técnica de investigación central para el estudio de las tecnologías en las Administraciones locales. En segundo lugar, se ofrece parte de los resultados de una investigación realizada por el autor sobre el desarrollo de la e-Administración en los Ayuntamientos de la Comunidad de Madrid, teniendo en cuenta la aplicación de un cuestionario. En tercer lugar, se realizan una serie de reflexiones sobre el uso del cuestionario en el análisis de la eAdministración local. Finalmente, se hace una breve conclusión que resume los contenidos más importantes del artículo.

\section{LAS TECNOLOGÍAS EN LAS ADMINISTRACIONES LOCALES}

El cuestionario es una herramienta de investigación ampliamente utilizada en el ámbito de las ciencias sociales, políticas y administrativas con una importancia comparable a otro tipo de técnicas de recogida de datos tanto de carácter cualitativo como cuantitativo. Las investigaciones sociales, políticas y administrativas que utilizan los cuestionarios han ido aumentando desde la consolidación de la corriente conductista-racional en los años 1960, de manera que muchos estudios académicos desde entonces se han apoyado en ellos por su capacidad para recoger información sobre un número amplio de unidades muestrales de análisis, usualmente en una coyuntura temporal concreta, con la intención de obtener de forma 
sistemática un cuerpo de datos cuantificable, respecto de un número de variables que se examinan para establecer patrones de asociación entre ellas (ANDUIZA, CRespo y MÉNDEZ, 1999). Desde luego, los estudios organizativos y, entre ellos, los que se centran en las Administraciones Públicas también han asumido de manera extensiva esta técnica de recogida de datos.

El cuestionario se ha utilizado de manera general como herramienta para mejorar el carácter empírico de las investigaciones en las que se ha aplicado. Los cuestionarios se han incluido en los diseños de investigaciones con diferentes unidades (o niveles) de análisis, bien los individuos dentro de un país a los que se realiza una encuesta electoral, bien las empresas de un determinado sector como el biotecnológico sobre las que se desea conocer sus prácticas de recursos humanos. Todas esas investigaciones han ido en paralelo con el aumento de la aceptación de publicaciones de carácter cuantitativo dentro de las revistas académicas de las ciencias sociales, políticas y administrativas. De hecho, los términos «sistemático» $\mathrm{y}$ 《cuantificable» tienen estrecha relación con el cuestionario, ya que esta técnica de recogida de datos se suele aplicar para obtener de la forma más precisa posible un dibujo cuantitativo de las unidades de análisis estudiadas, de ahí que se haya asociado fundamentalmente con la investigación empírica cuantitativa (AzofRA, 1999).

En el plano concreto de las organizaciones, numerosos autores han elaborado investigaciones basadas en la aplicación de cuestionarios. Dentro de los sectores más diversos, teniendo en cuenta diferentes tamaños u otras características organizativas se han propuesto diferentes tipos de diseños de investigación que tienen a las propias organizaciones o los individuos que trabajan en ellas como unidades de análisis, siendo el cuestionario la herramienta principal de recogida de datos (BRYMAN, 1995). La notable difusión de este tipo de trabajos ha llevado a que las Administraciones Públicas también se hayan convertido en un ámbito privilegiado para el uso de este tipo de técnica de investigación, de cara a descubrir patrones comunes de actuación, percepciones de sus miembros $\mathrm{u}$ otros aspectos de interés.

Una de las dimensiones de las Administraciones Públicas que tradicionalmente se ha analizado gracias a los cuestionarios ha sido su componente tecnológico, más recientemente, las TIC. La tecnología ha sido un tema central de investigación política desde hace al menos doscientos años (WINNER, 1977), de manera que no es de extrañar que se haya convertido en uno de los objetos de estudio concretos que ha merecido la atención de los investigadores dentro del área de Administración Pública 
en los que se ha aplicado el cuestionario como técnica de recogida de datos. La tradición cuantitativista de las ciencias sociales, generalmente asociada a las universidades estadounidenses, llevó a que muchos de sus académicos utilizaran los cuestionarios para analizar las primeras etapas de la difusión de los sistemas de información o los resultados de la implantación de los ordenadores personales en las Administraciones Públicas a partir de los años 1970, aunque es todavía más interesante el hecho de que muchos de estos estudios seminales se orientaran a las Administraciones locales (DANZIGer y DutTon, 1977; Dutton y KraeMER, 1978; KRAEMER, 1971).

Durante las tres últimas décadas han aparecido diferentes publicaciones sobre la difusión de las últimas tecnologías de información y comunicación en las Administraciones locales reflejando la capacidad de los cuestionarios para explorar esta realidad. Uno de los proyectos de investigación pioneros fue URBIS (Urban Information Systems) vinculado al PPRORG (Public Policy Research Organization Research Group) de la University of California, Irvine, que pretendió conocer el estado del arte de la computación/informatización en las Administraciones locales estadounidenses para evaluar el impacto que habían tenido los ordenadores en los servicios públicos y en la gestión del proceso de toma de decisiones, de manera que muchos de los análisis realizados se basaron en la aplicación de cuestionarios (DANZIGER et alia, 1982; DANZIGER y KRAEMER, 1986). La publicación de estos trabajos apareció inicialmente en revistas de marcado carácter tecnológico, como Communications of the ACM, MIS Quarterly o Urban Systems, pero también se abrió un hueco en otras del ámbito de Administración Pública como Administration \& Society, Policy \& Politics o Public Administration Review, dando origen varios años después (1991) a una publicación híbrida como Informatization and the Public Sector ${ }^{1}$.

Posteriormente, se han hecho diferentes investigaciones sobre las tecnologías en las Administraciones locales que han seguido muchas de las propuestas originales del proyecto URBIS y han puesto de relieve la importancia del cuestionario para analizar este tipo de objeto de estudio. En DANZIGER et alia (1993) se presentó un estudio sobre los factores que determinan la calidad de los servicios informáticos entre los usuarios

\footnotetext{
${ }^{1}$ Más tarde cambió su nombre por Information Infrastructure and Policy y desde 2002 se recupera como Information Polity. Más recientemente están apareciendo las primeras revistas específicas sobre e-Government: Electronic Journal of e-Government, International Journal of Electronic Government Research o Journal of e-Government, que dan cuenta de diferentes aspectos de la actividad administrativa relacionada con las tecnologías de información y las redes de comunicaciones.
} 
internos mediante un cuestionario realizado a casi dos mil de ellos (1.869) en las Administraciones locales de 46 ciudades estadounidenses. Para ello, los autores examinaron tres factores que la literatura considera que pueden influir en la calidad de los servicios informáticos, es decir, la estructura de la provisión del servicio, el nivel de los problemas tecnológicos y la orientación de los especialistas del servicio informático².

KING y KRAEMER (1991) utilizaron datos longitudinales del proyecto original (URBIS) y otros más recientes para adentrarse en porqué los sistemas de información van mejorando en las Administraciones locales, si las experiencias pasadas de uso marcan senderos de dependencia para el futuro o cómo pueden los directivos locales dar forma al desarrollo de esos sistemas de información. Este estudio se basó en un análisis de datos teniendo en cuenta diferentes olas de recogida sistemática mediante cuestionario a lo largo de varias décadas sobre la implantación, gestión, uso y efectos de la tecnología de información en más de 400 Administraciones locales ${ }^{3}$.

Por su parte, NORRIS (1999) sistematizó las aportaciones de diferentes investigaciones previas basadas en cuestionarios (Kramer y NoRRIS, 19964; ICMA, 1993, 1997; NORTHROP et alia, 1990) para profundizar en si las Administraciones locales utilizan de manera rutinaria las tecnologías de información y comunicación en sus actividades corrientes. Para tra-

\footnotetext{
${ }^{2}$ Los resultados del estudio no apoyaron el argumento popular según el cual los factores estructurales (por ejemplo, si los servicios informáticos están centralizados o descentralizados dentro de la organización) son los más importantes; tanto el nivel de los problemas operativos como las actitudes de los proveedores internos del servicio son más significativos. Los hallazgos apoyaron especialmente explicaciones centradas en factores de orientación del servicio, lo que llevó a los autores a subrayar la necesidad de que las estrategias para mejorar el servicio informático de las organizaciones se orientaran hacia la «interfaz sociotécnica» entre usuarios finales y proveedores de servicios internos.

${ }^{3}$ Los autores pusieron de manifiesto una serie de conclusiones sobre las tecnologías de información en las Administraciones Locales. Además de demostrar que las inversiones en infraestructura de tecnologías de información proporcionan grandes beneficios a largo plazo, también pusieron de manifiesto otros aspectos como la necesidad de gestionar el liderazgo, las razones para incrementar el uso de los sistemas de información, así como las estrategias más oportunas para llevar a cabo el crecimiento de esas potencialidades dentro del sector público local.
}

${ }^{4}$ En esta investigación se utilizó un cuestionario enviado a 7.135 Administraciones locales estadounidenses, de las que respondieron 2.327 (35\%). El objeto de la investigación consistió en conocer el alcance de la ayuda prestada por los ordenadores personales (PCs) a la gestión pública local, por lo que se comparó aquellas ciudadanes que sólo usaban PCs en su gestión con aquellas otras que también mantenían sistemas informáticos centrales. La conclusión principal fue que el uso de PCs dentro de las organizaciones analizadas no aceleraba la automatización de las funciones de gestión, de hecho sus usuarios estaban menos satisfechos, realizaban un uso menor de las tecnologías en general y era menos probable que utilizaran otro tipo de últimas tecnologías. 
tar esta cuestión, el autor realizó un análisis descriptivo y comparado de la extensión y profundidad de la difusión de una serie de tecnologías de información tradicionales y de última generación dentro de un grupo de varios cientos de Administraciones locales. Las primeras tenían automatizadas la mayoría de las tareas rutinarias (procesador de textos, bases de datos, presentaciones, etc., lo que indica extensión) en las diferentes áreas de gestión municipal (hacienda, recursos humanos, etc., lo que supone profundidad). Sin embargo, las segundas, las últimas tecnologías más complejas y vinculadas con la comunicación, no habían penetrado ni en términos de extensión, ni profundidad, con la misma intensidad dentro de las Administraciones locales analizadas ${ }^{5}$.

Recientemente, se ha seguido utilizando el cuestionario para conocer más sobre la difusión de las últimas TIC e Internet, en especial las páginas web, en las Administraciones locales. Por poner sólo dos ejemplos notables, MooN (2002) planteó una investigación sobre las características de las Administraciones locales estadounidenses que han adoptado una página web, el nivel de desarrollo de sus sitios en Internet en cuanto a la prestación de información y la provisión de servicios públicos y los factores que explican las variaciones encontradas. Este trabajo estuvo basado en un cuestionario aplicado por la International City/County Management Association en colaboración con una consultora y fue respondido por casi 1.500 Administraciones locales estadounidenses ubicadas en ciudades de más de 10.000 habitantes ${ }^{6}$.

Por su parte, Ho (2002) profundizó en la hipótesis del cambio de paradigma en la gestión de las Administraciones Públicas mediante un estudio sobre el desarrollo del e-Government local. Este trabajo se basó en una clasificación del desarrollo de la dimensión electrónica de las Administraciones locales analizadas en términos de integración de los contenidos de las

\footnotetext{
${ }^{5}$ A pesar de estas conclusiones, en parte derivadas de la novedad de tecnologías como las páginas web, los sistemas de información geográfica, el $e$-correo, etc., el autor planteó que, sobre todo, las últimas redes de comunicaciones producirán impactos positivos en las organizaciones, por lo que cada vez un mayor número de ellas las adoptarán y usarán, en un patrón de curva ascendente de aprendizaje. Además, si la penetración se extiende y se hace profunda, los beneficios serán más amplios y, finalmente, el hecho de que se adopten tecnologías de información y redes de comuncaciones más complejas supone que la integración y apoyo para ellas se convertirá en algo cada vez más crítico.

${ }^{6}$ El estudio demostró que el tamaño de las organizaciones y su tipo de gobierno son factores institucionales significativos que explican la implantación y desarrollo de la $e$-Administración local. Por otro lado, también se demostró que las lagunas técnicas, económicas y humanas se perciben como las mayores barreras para el desarrollo de la e-Administración en numerosas organizaciones locales $\mathrm{y}$, en general, muchas de ellas se encuentran en un estadio inicial de desarrollo y, aunque la adopción de páginas web sea muy elevada, todavía quedan por resolver determinados problemas institucionales que permitan exprimir todo su potencial.
} 
páginas web en diferentes paradigmas según su orientación administrativa, informacional o hacia el usuario, teniendo además en cuenta las percepciones de sus webmasters. Este trabajo tuvo como eje central un cuestionario aplicado a los responsables de la gestión de las páginas web de las Administraciones locales de las 55 ciudades más pobladas de los EE. UU., de manera que se contrastaron una serie de variables institucionales, socioeconómicas, gerenciales, etc., como potenciales factores explicativos de la adopción de un determinado paradigma en las páginas web observadas ${ }^{7}$.

En el caso de España, sólo recientemente se ha desarrollado algún ejemplo en torno a la medición de la difusión de las tecnologías en las Administraciones locales mediante cuestionario. Como caso más relevante se pueden citar los informes IRIA sobre la situación de la informática en las Administraciones regionales y locales españolas, realizados cada dos años desde principios de la década de 1990 por el Consejo Superior de la Informática y para el Impulso de la Administración Electrónica, órgano que se encarga de la preparación, elaboración, desarrollo y aplicación de la política informática del Gobierno central (CRIADO, 2003). En su aplicación a las Administraciones locales estos cuestionarios se han basado en una muestra estratificada de más de 500 Ayuntamientos españoles seleccionados en función de su tamaño y situación geográfica para establecer conclusiones sobre determinadas dimensiones de manera agregada y por tamaños, por ejemplo, las características del parque informático, los equipos disponibles, el gasto realizado en infraestructura informática, el tipo de software utilizado o el personal al servicio de esta nueva función de la gestión pública ${ }^{8}$.

La Fundación Retevisión (2004) también ha contado con datos procedentes de un cuestionario para realizar una serie de conclusiones sobre el

\footnotetext{
${ }^{7}$ El autor planteó que el nuevo paradigma que se une a la $e$-Administración, identificado con la construcción de redes coordinadas, la colaboración externa y los servicios públicos electrónicos integrados contradice el paradigma burocrático tradicional que enfatiza la estandarización, la departamentalización y la división del trabajo. La principal conclusión fue que muchas Administraciones locales estaban dando todavía los primeros pasos en esa nueva dirección, sin embargo, existen determinados factores socio-económicos e institucionales que inhiben la difusión del nuevo paradigma, tal es el caso de un número insuficiente de empleados dedicados a esta nueva función, las carencias en la financiación y el problema de la desigualdad digital unido a determinados sectores sociales.

${ }^{8}$ Los informes IRIA se basan en un exhaustivo cuestionario remitido a los responsables de la gestión tecnológica de los Ayuntamientos españoles, aunque ha sufrido una clara evolución. En la primera publicación de resultados se planteó que «el conocimiento de la infraestructura de medios técnicos y humanos en el área informática administrativa desempeña un papel fundamental y constituye un punto de partida necesario en el desarrollo de una verdadera estrategia de gestión y uso de las tecnologías de la información y las comunicaciones en las Administraciones Públicas» (MAP, 1993). Ahora se está pasando a dar mayor importancia a cuestiones que van más allá de la infraestructura tecnológica disponible y se adentran en la atención al ciudadano, sobre todo, mediante la
} 
uso de las TIC en los Ayuntamientos españoles desde 2001. En este caso, la muestra de Ayuntamientos también se ha realizado en función de los tamaños y distribución geográfica de los municipios y el objeto de los informes se ha ido depurando progresivamente, aunque el interés consiste también en analizar diferentes dimensiones relacionadas con la difusión de determinadas tecnologías, el impacto de las TIC en las Administraciones locales o las principales barreras para su difusión en el ámbito local, teniendo muy en cuenta las diferencias regionales que se producen en la agregación de los resultados por Comunidades autónomas?.

Más allá de los estudios realizados en el pasado, el interés de este artículo consiste en profundizar en las potencialidades específicas del cuestionario como técnica de recogida de datos para medir diferentes dimensiones de la $e$-Administración local $\mathrm{y}$, en concreto, en su posible aplicación dentro de realidades político-administrativas como la española. En las siguientes páginas se presenta una investigación aplicada en la que se han tenido en cuenta los precedentes citados, abordando la realidad de las tecnologías en el conjunto Administraciones locales de una región española.

\section{UN ANÁLISIS DE LOS AYUNTAMIENTOS DE LA COMUNIDAD DE MADRID}

En esta sección se desarrolla un ejemplo de aplicación del cuestionario en el estudio de la $e$-Administración local en una región española. Las siguientes páginas ofrecen parte de los resultados de una amplia investi-

\footnotetext{
provisión de información y la prestación de servicios electrónicos, de manera que en el último informe publicado se decía que si bien «el Informe IRIA se ha consolidado como un importante elemento de difusión y ayuda a la gestión en el campo de los sistemas y tecnologías de la información en el ámbito administrativo, inmersos como estamos en el proceso de implantación de la Administración electrónica y el desarrollo de servicios on line a los ciudadanos se hace necesario conocer la evolución de este fenómeno, contar con datos fiables que permitan analizar situaciones anómalas $y$ adoptar medidas correctoras para paliar las mismas» (MAP, 2002).

9 En los últimos años, la presencia de los Gobiernos y Administraciones Públicas en Internet dependía de la iniciativa, a veces personal, de los políticos, directivos y empleados públicos por promover la presencia de su organización en la Red. Sin embargo, la Ley 57/2003 de Medidas para la Modernización del Gobierno Local establece en su artículo 70 bis, apartado 3 que «las Entidades locales, y especialmente los municipios, deberán impulsar la utilización interactiva de las TIC para facilitar la participación y la comunicación con los vecinos, para la presentación de documentos y para la realización de trámites administrativos, de encuestas y, en su caso, de consultas ciudadanas. Las Diputaciones provinciales, Cabildos y Consejos insulares colaborarán con los municipios que, por su insuficiente capacidad económica y de gestión, no puedan desarrollar en grado suficiente el deber establecido en este apartado». De ahí el interés creciente que suscita la difusión e impacto de las Tecnologías de la Información y la Comunicación (TIC) e Internet en el ámbito político-administrativo local español (Criado y Ramilo, 2003b).
} 
gación sobre la e-Administración local en España, tomando como casos de estudio los municipios con más de 2000 habitantes de la Comunidad de Madrid ${ }^{10}$. Además del propio valor intrínseco de los datos que se ofrecen, en este artículo se pone un especial acento en la reflexión sobre el uso del cuestionario como herramienta de investigación para mejorar la medición y el análisis de las dimensiones de la $e$-Administración, en general, y la profundización en diferentes aspectos relacionados con las tecnologías en las Administraciones locales, en particular.

\section{Diseño de la investigación}

Este estudio partió de una revisión de los trabajos existentes sobre las tecnologías en las Administraciones locales, en especial, teniendo en cuenta la realidad española. El objetivo de la investigación consistió en recoger de manera sistemática datos primarios sobre la situación de la $e$ Administración dentro del nivel local en una región española, dibujando una foto fija acerca de varias dimensiones de interés dentro de un ámbito de estudio en el que existía una notable escasez de información. Todo ello, por encargo de la Federación de Municipios de Madrid, organismo público que avaló la realización del trabajo de campo, aunque el perfil del equipo de investigación era netamente académico, así como toda la investigación en su conjunto.

Las preguntas de la investigación cubrían un amplio espectro de intereses, dado el interés del estudio para un público potencialmente diverso. Algunas de esas preguntas fueron las siguientes: ¿existe una planificación que oriente la difusión de la $e$-Administración en el sector público local? ¿Cuál es el nivel de difusión de las tecnologías de información y comunicación entre las Administraciones locales? ¿Las últimas tecnologías de información y comunicación se han vinculado a cambios de calado en diferentes dimensiones de la gestión y la comunicación con el entorno? ¿Por qué adoptan páginas web las Administraciones locales? ¿Cuáles son las ventajas de su uso? ¿Cuáles son las barreras que encuentra la e-Administración en el sector público local? Desde un primer momento, se fue consciente de que muchas de las respuestas a estos interrogantes quedarían sin una respuesta definitiva

\footnotetext{
${ }^{10}$ Esta sección recoge parte de los planteamientos y datos de una publicación del autor (Criado, 2004a). Esta monografía recibió en diciembre de 2003 una Mención Especial en el III Premio Fermín Abella y Blave para trabajos de estudio e investigación sobre la Reforma Administrativa en la Administración Local del Instituto Nacional de Administración Pública, Ministerio de Administraciones Públicas.
} 
como consecuencia del propio alcance de la investigación, aunque se llevara a cabo un análisis descriptivo que podría ofrecer claves para trabajos futuros.

Teniendo en cuenta esas preguntas, se planteó que la mejor manera de llevar a efecto la investigación suponía adoptar como una de las técnicas de recogida de datos principales el cuestionario. El cuestionario fue diseñado con preguntas sobre una serie de aspectos cuantitativos de hecho y percepciones relacionadas con las últimas tecnologías de información y comunicación e Internet dentro de los Ayuntamientos, dividiéndose en cuatro partes: un bloque de preguntas sobre los recursos generales en TIC disponibles; otro sobre los principales usos que están haciendo de las TIC dentro de algunas de sus principales áreas de actuación; un tercero en que se hacían preguntas específicas acerca de las páginas web, en los casos en los que existían; finalmente, se pretendía recoger información sobre las barreras más importantes a la adopción de la $e$-Administración por parte de los Ayuntamientos.

El trabajo de campo relacionado con el cuestionario se extendió desde el 15 de diciembre de 2002 al 1 de marzo de 2003. El cuestionario fue remitido directamente a los Alcaldes de los 179 Ayuntamientos madrileños, aunque los destinatarios finales eran los responsables de las unidades encargadas de la gestión de las tecnologías, en aquellos con personal de este tipo, o las personas más próximas a ese perfil. Los cargos que finalmente respondieron los cuestionarios fueron los jefes de áreas de Informática, Nuevas Tecnologías, Sistemas de Información, Comunicaciones, Innovación Tecnológica, etc., y sólo en algún caso los completaron cargos diferentes como Agente de Desarrollo Local o Agente de Innovación Tecnológica. No obstante, uno de los problemas de la variedad de temas incluidos en el cuestionario, y el hecho de no suministrarlo personalmente sino vía postal o por correo electrónico, fue que los encuestados tuvieron ciertas dificultades para recopilar la abundante información solicitada para rellenarlo correctamente, sobre todo, si la información estaba dispersa en diferentes unidades administrativas dentro de determinados Ayuntamientos.

La presentación de los resultados cuantitativos del estudio se detalló a través de un método estadístico descriptivo utilizando frecuencias simples de aparición o medias aritméticas de cada uno de los indicadores, aunque las características específicas de las dimensiones analizadas en cada pregunta se concretan después. Por otra parte, para algunas cuestiones específicas se utilizó un análisis cualitativo de los datos, puesto que se trataba de preguntas abiertas en los cuestionarios, en concreto, respecto a las 
percepciones sobre las estrategias de $e$-Administración de los Ayuntamientos ${ }^{11}$.

\section{Algunos datos de partida}

La primera sección del cuestionario pretendía obtener una serie de datos básicos sobre las Tecnologías de Información y Comunicación (TIC) e Internet en los Ayuntamientos de la Comunidad de Madrid. Por un lado, se profundizó en el nivel de difusión de determinadas redes y tecnologías y, por otro, en el alcance de las planificaciones sobre $e$-Administración dentro de los Ayuntamientos. Inicialmente, cabe decir que sobre el total de organizaciones objeto de estudio (92), 45 remitieron el cuestionario completado, lo que supone que la muestra analizada se refiere a casi un 50\% del conjunto dentro del estrato de población propuesto (los Ayuntamientos de más de 2.000 habitantes de la región), lo que aseguró el nivel de representatividad de los datos disponibles ${ }^{12}$.

Un primer aspecto general que resultaba interesante conocer se refiere a la disponibilidad de uso de determinadas redes y servicios asociados a las TIC ampliamente extendidos en las organizaciones del sector privado. En este caso, se dispone de información sobre el grado de adopción en el conjunto de Ayuntamientos analizados de Redes de área local (RAL), Intranets, acceso a Internet y página web. En general, se puede afirmar que el acceso a Internet se ha universalizado en los Ayuntamientos $(100 \%)$ y que las páginas web $(91.1 \%)$ y las $R A L(93.3 \%)$ están presentes en un amplio porcentaje de ellos. Por su parte, las Intranet han llegado a un número más limitado de organizaciones $(37.8 \%)$, quizá como consecuencia de la mayor complejidad técnica para desarrollar este tipo de herramienta de comunicación interna. No obstante, más allá de los porcentajes concretos de adopción, lo interesante es realizar una constatación del amplio nivel de presencia dentro de las Administraciones locales de este tipo de redes y tecnologías. No sorprende el acceso masivo a la red

\footnotetext{
${ }^{11}$ Para una contextualización más amplia de la investigación o para completar los datos aquí disponibles, se recomienda la consulta de la obra citada antes.

${ }^{12}$ La Comunidad de Madrid está distribuida en 179 municipios. Se utilizó un universo de Ayuntamientos más homogéneo en cuanto a tamaño y limitado en número para facilitar el establecimiento de tendencias compartidas, sin los problemas del inframunicipalismo, muy habitual en España. Por ello, las conclusiones de esta investigación se deben limitar a Administraciones locales con una capacidad institucional mínima para gestionar sus recursos tecnológicos, que aquí se ha planteado en el umbral de población de 2.000 habitantes, ya que a partir de este tamaño los Ayuntamientos empiezan a contar con personal dedicado a esta labor. En todo caso, al final se plantean los problemas para generalizar este tipo de observaciones.
} 
Internet en los Ayuntamientos españoles, observado en otras investigaciones anteriores, lo que demuestra un alto grado de penetración en las Administraciones locales en consonancia con su creciente aceptación social.

Esta primera parte del cuestionario incorporaba un apartado de carácter cualitativo que pretendía ofrecer una panorámica general sobre la presencia y los contenidos de los planes generales de e-Administración de los Ayuntamientos. En este caso se deseaba conocer el nivel de adopción de programas específicos sobre uso de las TIC e Internet dentro de las Administraciones locales, así como una definición de sus planteamientos estratégicos. Por un lado, los resultados son escasos en términos generales, ya que sobre el conjunto de Ayuntamientos, menos de una cuarta parte de ellos $(24.4 \%)$ ha introducido una planificación de estas características. En los casos en los que sí se ha implantado de forma estratégica un programa de $e$-Administración se preguntaba de manera abierta por sus objetivos principales. En general, se han detectado una serie de puntos críticos reiterados en las respuestas de los gestores TIC de los Ayuntamientos, que conformarían un núcleo de objetivos básicos de las estrategias de $e$-Administración local:

- la disponibilidad para los ciudadanos de información y servicios en línea,

- el acercamiento del Ayuntamiento a los ciudadanos mediante la mejora de la comunicación con el entorno,

- la mejora de la gestión interna, sobre todo, en lo que se refiere a capacidad de intensificar la comunicación entre organismos ubicados en espacios geográficos dispersos,

- la integración de soluciones y la unificación de sistemas y datos entre diferentes unidades administrativas,

- la automatización de los procedimientos administrativos en diferentes ámbitos de gestión y,

- la optimización de los costes de los recursos técnicos y humanos utilizados.

Se observó que un núcleo pequeño de Ayuntamientos, sobre todo los de mayor tamaño, tienen ideas consolidadas sobre cuáles tienen que ser los aspectos estratégicos de una propuesta general de e-Administración local. Como se esperaba, sus esfuerzos se orientan a mejorar la gestión interna y a abrir una nueva vía a la atención a los ciudadanos mediante la provi- 
sión de información y la prestación de servicios, fundamentalmente, vía web. Por otra parte, también se ha comprobado que las iniciativas relacionadas con la $e$-Administración, en general, son propuestas escasamente ambiciosas. En la gran mayoría de los casos, más bien se trata de medidas en las que destaca la falta de coherencia en su formulación y la escasa consideración de los problemas unidos al proceso de implementación.

Junto a lo anterior, también se solicitaba información sobre las medidas que los gerentes de los Ayuntamientos que carecen de planificación para el desarrollo de la e-Administración incluirían en iniciativas generales de este tipo. A objetivos citados por aquéllos, éstos unieron una serie de inquietudes complementarias, especialmente, la atención a la formación de los empleados públicos, la evaluación periódica mediante auditorías, la actualización permanente del equipamiento tecnológico disponible, el establecimiento de estándares de seguridad y privacidad en las relaciones con los ciudadanos y las empresas o la necesidad de generar consenso dentro de la organización en el proceso de implementación.

En resumen, existe un porcentaje limitado de Ayuntamientos que han observado la difusión de la $e$-Administración como estratégica y, por tanto, han plasmado su interés (más allá de la adopción de infraestructura básica) mediante una planificación general. A la luz del análisis cualitativo de los datos, y como ya se pusiera de manifiesto en estudios clásicos sobre las tecnologías en las Administraciones locales, muchas de las acciones concretas dentro de la dimensión TIC en este nivel de administración se realizan sin una reflexión a nivel estratégico sobre su calado político, ni sus consecuencias en la propia organización y la relación con los ciudadanos. A pesar de su contenido técnico, el problema de las tecnologías en las Administraciones Públicas es político, de manera que los políticos son los que han de marcar las líneas generales de incorporación de recursos, aunque las actuaciones sean consensuadas, facilitadas y concretadas a nivel técnico (RAMIÓ, 2002). La segunda parte del cuestionario pasaba a considerar la percepción de los gerentes TIC sobre la difusión e impacto de las tecnologías en diferentes ámbitos de sus organizaciones.

\section{Interacciones entre gestión pública local y e-Administración}

Junto al conocimiento de los recursos básicos citados, con el cuestionario se profundizaba en la difusión de las TIC e Internet dentro de diferentes ámbitos de la gestión y comunicación de las Administraciones locales. En general, se trataba de localizar la existencia o no de tendencias 
compartidas hacia una transformación efectiva de la gestión, la prestación de servicios sectoriales o las relaciones con actores del entorno gracias a la utilización de las TIC e Internet por parte de los Ayuntamientos. Como se puso de relieve en numerosas entrevistas informales desarrolladas a lo largo del estudio, los responsables directivos de las TIC en las Administraciones locales cuentan con una visión muy completa sobre sus organizaciones, tanto en lo concerniente con las tecnologías como sobre diferentes ámbitos de la gestión pública local, debido a la horizontalidad de su función.

La primera dimensión planteada en los proyectos de $e$-Administración como susceptible de transformación se refiere a la gestión interna. En este caso, se deseaba conocer en qué medida las TIC e Internet han transformado determinados ámbitos de la gestión interna de los Ayuntamientos analizados, de manera que se preguntó a los directivos por el nivel de impacto (nada (1), poco (2), bastante (3) o mucho (4)) de las tecnologías en determinadas funciones. Existe una opinión compartida bastante positiva, aunque no revolucionaria, sobre el impacto de las TIC e Internet dentro de la gestión interna de los Ayuntamientos. En efecto, destacan una serie de funciones clave de la gestión pública local en las que el nivel de aplicación de las TIC e Internet resulta muy apreciable, sobre todo, la gestión económico-financiera, gestión de recursos humanos y capacidad de control. Otro grupo sólo se acerca de lejos a ese nivel, la coordinación entre unidades administrativas y la comunicación interna. Finalmente, dos aspectos concretos como la gestión de proyectos o la gestión documental se han visto afectados en menor medida por las tecnologías, a pesar de que tienen una importancia creciente dentro de las organizaciones públicas.

Más allá de los resultados específicos por funciones, parece que las TIC e Internet se han aplicado más a los ámbitos de gestión relacionados con la posibilidad de obtener, almacenar y distribuir información dentro de las organizaciones (la I de las TIC), que a los que ofrecen la capacidad de mejorar la comunicación dentro de ellas (la $\mathrm{C}$ de las TIC). Esto viene a confirmar la idea de que, todavía hoy, los principales usos de las tecnologías en las Administraciones Públicas se suelen orientar más a la mejora de la eficiencia de la gestión que a la comunicación interna o con el entorno, como se comprueba después. Además, a pesar de que los resultados descartan una tendencia hacia transformaciones radicales en la gestión interna de las Administraciones locales, sí muestran una serie de ámbitos de interés compartidos en los que la aplicación de las tecnologías ha sido mucho más que modesta. 
En segundo lugar, se presentan conclusiones sobre la difusión de las TIC e Internet en la prestación de servicios sectoriales. La importancia de esta dimensión reside, no sólo en que los informes que pretenden medir el desarrollo de la e-Administración evalúan el nivel de prestación de determinados servicios públicos mediante páginas web, sino también en la repercusión interna de las TIC e Internet en esa función de prestación con carácter general, y no sólo a través de aquéllas. Este hecho se asienta en la realidad de que los Ayuntamientos han asumido cada vez más competencias en diferentes dominios de las políticas públicas en el caso español. Se tuvo en cuenta la división de los servicios públicos entre aquellos de carácter personal, como educación, desarrollo económico, salud, etc., y urbanos, por ejemplo, medio ambiente, obras, urbanismo, tráfico, etc., siguiendo la misma escala antes utilizada (nada (1), poco (2), bastante (3) о тисьо (4)).

En general, dentro de esta dimensión se ha manifestado un nivel de impacto apreciable, aunque existen unas diferencias muy significativas entre unos servicios y otros y, en general, la intensidad es menor que los valores mostrados en la dimensión anterior. Primero, no es igual el uso de las TIC en la prestación de servicios como urbanismo, desarrollo económico o policía, que tienen un calado político mayor, que en los servicios relacionados con el turismo, transporte público o limpieza, en los que su aplicación es muy limitada. Como se decía, existe una serie de servicios públicos en los que los gestores han observado una difusión muy considerable de las TIC, especialmente, urbanismo, desarrollo económico, policía, deportes, empleo, cultura y servicios sociales, que son algunas de las áreas de acción política más relevantes de los Ayuntamientos españoles. Segundo, las Administraciones locales suelen hacer un uso más intensivo de las TIC e Internet en los servicios de carácter personal, es decir, aquellos orientados directamente a personas, que en los servicios de carácter urbano. Ambos aspectos confirman que la propia naturaleza de los servicios públicos tiene algo que ver con las posibilidades para la utilización más o menos intensiva de las tecnologías en su producción interna o para su completa digitalización.

En conclusión, la prestación de servicios públicos se está viendo transformada, aunque de forma limitada, como consecuencia de la incorporación de las TIC en su producción. En este sentido, las páginas web están incrementando las posibilidades de las Administraciones locales de cara a ofrecer una nueva vía para las funciones de provisión. No obstante, y a pesar de las promesas, todavía existe una percepción generalizada de que los cambios se están orientando en mayor medida al núcleo interno de gestión de las organizaciones, que a la prestación directa de servicios 
públicos a los ciudadanos, en otras palabras, se insiste en la idea de que se está buscando la mejora de la eficiencia interna por encima de la satisfacción de los ciudadanos, aunque estos sean los destinatarios finales de los resultados de la acción gubernamental.

En tercer lugar, el cuestionario interrogó a los gerentes acerca de su percepción sobre el impacto de las TIC e Internet en la comunicación con otros actores del entorno. Sin duda, esta dimensión es la que presenta niveles más discretos, lo que vuelve a insistir en la necesidad de rechazar cambios revolucionarios asociados a la e-Administración local, y menos todavía teniendo en cuenta el impacto global de las TIC e Internet sobre la capacidad de mejora de la comunicación de las organizaciones. En esta dimensión también se planteó una escala semejante a las anteriores (nada (1), poco (2), bastante (3) o mucho (4)), atendiendo a las percepciones sobre el nivel de impacto en la comunicación con actores del entorno organizativo.

Esta dimensión ofreció unos resultados con menores diferencias entre las categorías formuladas, sin embargo, en conjunto reflejaron unos niveles más limitados de cambio que las otras dos dimensiones analizadas previamente. Primero, a pesar de lo que se pudiera pensar a priori, se ha observado una mejora mayor de la comunicación con otras Administraciones Públicas que, por ejemplo, respecto a otros actores del entorno más próximo, como las asociaciones del municipio u otros agentes municipales como empresas, sindicatos, etc. Segundo, entre las Administraciones Públicas destaca un impacto mayor en la comunicación con las Administraciones regional, central y locales, que con la Administración de la UE, que se percibe más alejada y con la que parece que la interlocución se hace sólo un poco más efectiva gracias a las TIC e Internet. Finalmente, las tecnologías han tenido una incidencia menor en las comunicaciones con otros actores del ámbito local, lo que sucede tanto para los agentes municipales (sindicatos, organizaciones empresariales, etc.), como respecto de las asociaciones del municipio, bien sean culturales, juveniles o vecinales. En todo caso, otros entes internos del Ayuntamiento (empresas con participación pública, organismos autónomos, etc.) sí han logrado un alto nivel de mejora, avance fundamentalmente vinculado a la generalización del uso del correo electrónico para la comunicación con este tipo organismos que, a pesar de formar parte del Ayuntamiento, no se identifican con su estructura central y disponen de amplios márgenes de autonomía.

En suma, aquí se abre la puerta a una conclusión de carácter general: a pesar de las facilidades ofrecidas por las nuevas tecnologías, cada vez más 
orientadas a la comunicación externa, se aprecia una tendencia de uso hacia la mejora de la gestión interna de las Administraciones locales, por encima de su orientación hacia la innovación de las comunicaciones con el exterior. En futuras investigaciones habría que profundizar en las razones de este recelo a enlazar mejor las Administraciones locales con un entorno que, al menos de manera intuitiva, parece cada vez más accesible mediante un uso adecuado de los nuevos medios de comunicación disponibles.

\section{Motivaciones para la creación de páginas web y beneficios de su desarrollo}

Las Páginas web se han ido incorporando de forma progresiva a las Administraciones locales desde su origen a principios de la década de 1990. Aunque todavía no existe un análisis detallado de este fenómeno, las páginas web se han convertido en la innovación tecnológica más popular que han adoptado las Administraciones Públicas, en general, y las Administraciones locales, en particular, en las últimas décadas (CRIADO y RAMILO, 2001). Los estudios que se han centrado en esta tecnología de forma específica destacan su capacidad para convertirse en un nuevo canal de comunicación con los ciudadanos y las empresas, capaz de competir y superar al presencial y telefónico (LA PoRTE, DEMCHACK y de Jong, 2002; Musso, Weare y Hale, 2000; Scavo y Yuhang, 1999), aunque no deje de ser complementario mientras las tasas de penetración de Internet sean tan limitadas en muchos países. Aunque su importancia todavía es relativa dentro de las Administraciones locales, las páginas web se han incorporado de manera rápida en el ámbito local español, así como en los Ayuntamientos de la Comunidad de Madrid (CRIADO y RAmiLo, 2003a). Dentro de este apartado, se pretendía recoger la opinión de los gestores TIC de los Ayuntamientos sobre dos aspectos relacionados con sus páginas web: las razones que motivaron su creación y sus principales ventajas de uso.

En lo que respecta a los motivos para la adopción de una página web, en general, se trataba de conocer qué tipo de razones impulsaron a las Administraciones locales a acelerar el proceso de incorporación de una página web como parte de su estructura organizativa $\mathrm{y}$, de paso, conocer cuáles fueron las potencialidades y beneficios que observaron en esta tecnología. El cuestionario ofrecía ocho opciones cerradas y se pidió que se seleccionaran tres. Lo primero que se puede apuntar es que existe una amplia variedad en las respuestas ofrecidas, dado que una mayoría considerable de las posibilidades cuenta con más de un $10 \%$ de presencia. De 
hecho no se descarta ninguna opción, aunque su concreción sea menor, tal es el caso de potenciar el turismo del municipio (6.1\%). La razón que los Ayuntamientos consideran como principal para explicar la adopción de una página web es la de establecer un nuevo canal de comunicación del Ayuntamiento con el ciudadano (24.3\%) y apuntan la necesidad de modernizar el Ayuntamiento y la Administración municipal como segundo aspecto prioritario de explicación (15.7\%).

En estos Ayuntamientos suele existir una visión sólida sobre el significado de las TIC e Internet con carácter general. Los directivos conceden más importancia a las razones de creación relacionadas con lograr que el Ayuntamiento esté en la Red (14.8\%) y no quedar atrás con respecto al desarrollo de las TIC (11.3\%), que las opciones que se identifican con mejorar la imagen del Ayuntamiento o difundir los logros municipales (con el $10.4 \%$ y $7.8 \%$ de las respuestas, respectivamente), que tienen un carácter más político. Ese mayor peso relativo concedido a las TIC e Internet implica una atención a su propio valor intrínseco, esto es, al valor añadido que aportan a las actividades en las que se aplican de forma intensiva, por encima de la visión como medio o escaparate que, todavía hoy, mueve muchas de las acciones vinculadas a la adopción de una web pública.

La segunda cuestión sobre las páginas web pretendía conocer las principales ventajas asociadas al desarrollo de este tipo de tecnología en las Administraciones locales. Esta pregunta refleja una visión interesante acerca de los puntos positivos que los Ayuntamientos de la Comunidad de Madrid han observado durante el uso de sus páginas web. La pregunta se formulaba ofreciendo diez posibilidades cerradas sobre las que se podía elegir un máximo de tres. En este sentido, las ventajas planteadas se seleccionaron atendiendo, principalmente, a dos grandes ámbitos: la gestión interna y la atención a los ciudadanos.

Las dos principales ventajas de contar con una página web están unidas a la mejora de la atención a los ciudadanos. En este sentido, las respuestas que obtuvieron mayor apoyo se refieren a la posibilidad para el Ayuntamiento de establecer un nuevo canal de comunicación con el ciudadano $(24.1 \%)$, seguida del aumento de la capacidad para mejorar la información que disponen los ciudadanos (21.6\%). Ambas opciones muestran una visión optimista sobre el potencial de este tipo de tecnología, ya que se tiende a subrayar su importancia desde la perspectiva del ciudadano, que en este caso sí parece ser el destinatario final de las mejoras asociada a la $e$-Administración local. 
Las opciones sobre la mejora de la gestión interna fueron seleccionadas como ventajas en un menor número de casos. Dentro de este grupo se ha considerado la capacidad de mejora de la imagen del Ayuntamiento $(14.7 \%)$, que también cuenta con una clara proyección externa, en mayor medida que las posibilidades para modernizar el Ayuntamiento y la Administración municipal (12.9\%) y la mejora de la calidad de los servicios públicos (8.6\%), aunque la suma de estas tres categorías da una idea de sus ventajas añadidas de cara al desarrollo de una página web. Una presencia menor tienen otras cuestiones, como el aumento del turismo del municipio (5.2\%), así como otros aspectos relacionados con la gestión interna, tales como sistematizar la prestación del servicio de información $(5.2 \%)$, incrementar la productividad de los trabajadores o apoyar la coordinación y gestión internas (ambas con un 1.7\%).

En suma, este apartado puso de manifiesto que las páginas web muestran unas características específicas que aportan un nuevo valor añadido para las organizaciones públicas. Bien sea por sus reducidos costes o por su proyección externa, parece cada vez más claro que las páginas web se han convertido en un tipo de tecnología que se está orientando a facilitar la comunicación de las Administraciones locales con el entorno de una manera desconocida para otras tecnologías previas. En este punto, algunos autores perciben unas potencialidades muy significativas para el futuro de las Administraciones locales (MARGETTS y DUNLEAVY, 2002) y la amplia posibilidad que ofrecen muchos Ayuntamientos españoles para la prestación de servicios públicos electrónicos es un reflejo de ello.

\section{Barreras contra la $\boldsymbol{e}$-Administración local}

Finalmente, se consideraron algunos problemas, tanto internos como externos a las Administraciones locales, que sus directivos TIC plantean como inhibidores de la difusión de las TIC en ellas. La necesidad de conocer algo más sobre las barreras para extender la $e$-Administración local resulta de gran importancia de cara a delimitar con claridad la problemática de la institucionalización de las tecnologías. Junto con un conocimiento más profundo de la realidad político-administrativa en torno a las TIC, también resulta necesario plantear las barreras que impiden una mayor presencia en las Administraciones locales. En este caso, cada una de las preguntas formuladas ofrecía diez posibilidades cerradas de respuesta (más una categoría abierta con otras barreras), dando la opción para seleccionar tres propuestas. Como primer comentario general, la categoría otras barreras fue utilizada en una sola ocasión (refiriéndose a la escasez de estudios de referencia sobre este problema), por lo que se 
puede decir que los temas seleccionados apuntaron bastante bien la problemática general.

En lo que respecta a las barreras internas, había una serie de puntos críticos de carácter endógeno que se pretendía corroborar mediante las respuestas recibidas. Principalmente, se ha demostrado que los aspectos que los directivos TIC suelen considerar como críticos en la expansión de la $e$-Administración se refieren a los recursos materiales y humanos, los aspectos de carácter institucional y de cultura organizativa, así como problemas asociados al componente político de la toma de decisiones en el sector público.

Como se ha dicho, existe una preocupación generalizada por los recursos materiales y humanos relacionados con las TIC dentro de las Administraciones locales. En este sentido, dos de las posibilidades de respuesta asociadas con los recursos han superado el $20 \%$ de las respuestas. Por un lado, el problema de la carencia de recursos dentro del ámbito material ha sumado el $22.0 \%$, mientras que la falta de personal se ha situado por detrás con el 20.3\%. Estos datos confirman algo que algunos gestores públicos han corroborado en conversaciones informales: las carencias de personal y materiales son especialmente sensibles dentro de los departamentos relacionados con las TIC, aunque en algunos casos se puede hablar de carencia casi total de recursos propios, si se excluyen subvenciones y dotaciones procedentes de otras Administraciones Públicas, en las organizaciones de menor tamaño.

Por otra parte, las barreras institucionales también están claramente representadas en las opiniones de los gestores. De hecho, en tercer y cuarto lugar como barreras internas más importantes se han situado las respuestas relacionadas con aspectos de carácter institucional, siendo la falta de cultura informática en la organización $(16.1 \%)$ y la preocupación por la falta de formación de los empleados (11.0\%) dos claras muestras de inhibidores a la hora de institucionalizar las tecnologías. En este caso, se trata de barreras establecidas como consecuencia de particulares componentes culturales, valores, preparación, creencias que, en determinados contextos públicos, se ha demostrado suponen obstáculos a la innovación basada en las tecnologías.

Finalmente, otro grupo de barreras internas merece una menor preocupación global, aunque existe algún aspecto de especial interés. En primer lugar, la preocupación por los problemas de seguridad (8.5\%) pone de manifiesto la necesidad de dotarse de medidas de seguridad internas antes de realizar un intercambio con el exterior que ofrezca un mínimo de 
garantías. Otros aspectos que se han apuntado en menor medida son los problemas de coordinación administrativa (7.6\%), la rápida obsolescencia de los equipos $(7.6 \%)$ y la desconfianza de los empleados $(1,7 \%)$. El hecho de que este tema no es una prioridad política (5.1\%) no se ha considerado de manera abierta en los cuestionarios, sin embargo, hay que decir que muchos responsables técnicos, de manera privada, han mostrado una preocupación por las carencias en el liderazgo político, como un problema que no facilita la institucionalización de las tecnologías en las Administraciones locales. En ese sentido, no hay que obviar como factor determinante el apoyo de los responsables políticos para realizar inversiones materiales, para elaborar políticas de estabilización de recursos humanos y, sobre todo, para que el sector público local adopte una visión estratégica sobre la $e$-Administración, aspecto crítico que supone un verdadero reconocimiento de la naturaleza política de este tema.

El segundo grupo de barreras para la difusión de la e-Administración local se refiere a la vertiente externa a las organizaciones. Dentro de las barreras externas que normalmente se plantean como freno a la innovación tecnológica dentro del sector público se ha entendido que la influencia de los ciudadanos resulta decisiva. A las barreras humanas se han unido otros problemas específicos como la privacidad y la seguridad, las relaciones con otras organizaciones, así como la percepción de la tradición administrativa y las formas de relación de las Administraciones Públicas con los ciudadanos.

El principal factor mencionado por los directivos TIC es la desigualdad basada en la tecnología (22.9\%), es decir, la brecha digital. Esta cuestión pone sobre la mesa la preocupación por la escasa apropiación social de Internet, teniendo en cuenta las diferentes esferas en las que se puede manifestar su difusión. Aunque luego se profundiza más en este aspecto, aquí cabe decir que ha sido el problema más asumido entre todos los planteados. Dentro del resto de barreras externas también se ha formulado la falta de información de los ciudadanos (13.8\%). En este sentido, se trata de poner el acento sobre un problema clásico de la gestión pública, si se asume que la actividad pública está orientada a lograr la mayor equidad posible mediante la prestación de servicios y otras acciones desde las Administraciones Públicas. Sin embargo, las carencias de información sobre el sector público, precisamente, se suelen concentrar en aquellos que tienen menos estudios y renta. En ese sentido, Internet es un medio donde se reproducen esas desigualdades, puesto que es utilizado por aquellos con mayor formación y renta, de manera que una actuación pública que refuerce este canal, sin ampliar su difusión o sin potenciar los canales de información presencial y telefónico, estaría favore- 
ciendo, incluso sin desearlo, a aquellos con una mejor posición de partida en la sociedad.

Los problemas de seguridad y privacidad tienen también una incidencia cada vez más importante como barreras externas al desarrollo de la $e$ Administración local. Aquí se han alcanzado unos resultados considerables en las dos vertientes del tema: la seguridad (inseguridad de los ciudadanos, $15.6 \%$ ) y la privacidad (problemas de privacidad, 11.9\%). En ese sentido, existe una sensibilidad creciente entre los directivos TIC para mejorar la percepción externa sobre la fiabilidad de los canales digitales de comunicación con el sector público, mediante la persecución de los fraudes o las violaciones de la intimidad, a medida que se hace más intensivo el uso de las tecnologías en la intermediación y la prestación de servicios. Otras barreras externas ampliamente compartidas por los Ayuntamientos tienen un carácter más heterogéneo. Primero, se trata de las reticencias de los ciudadanos a perder el contacto personal con los funcionarios (10.1\%), lo que insiste en la necesidad de entender las TIC no como sustitutivo, sino como un canal complementario de comunicación, junto con el presencial y el telefónico. Además, la cuestión de la falta de implicación de otras Administraciones Públicas (10.1\%) supone la preocupación de las Administraciones locales por la colaboración intergubernamental, con especial interés en lo que respecta a compartir recursos humanos y materiales, promover normativa, desarrollar infraestructuras o lograr estándares para avanzar de manera conjunta con los municipios próximos o con características semejantes.

Otras barreras que se ofrecían como susceptibles de atención han merecido una consideración testimonial, tal es el caso de los problemas con los proveedores de software (3.7\%) y la ausencia de colaboración del sector privado $(0.9 \%)$. Parece que los Ayuntamientos consideran que los ciudadanos perciben un valor añadido en las actividades basadas en TIC, pero llama la atención que el sector privado quede al margen de la preocupación de los Ayuntamientos, puesto que la incapacidad para asumir riesgos impide en muchas ocasiones la extensión de determinadas infraestructuras en el ámbito rural o no permite asumir a las Administraciones locales determinados costes relacionados con las licencias de software.

En conclusión, estos resultados han permitido hacer un diagnóstico bastante fiable, aunque muy limitado, sobre algunos aspectos de las Administraciones locales de una región española en lo que se identifica como su componente electrónico. En síntesis, se puede afirmar que algunos Ayuntamientos de la Comunidad de Madrid poseen una situación evolucionada respecto de la e-Administración, aunque todavía quedan 
muchos puntos de mejora, teniendo en cuenta que una perspectiva madura referida a las TIC dentro de las Administraciones locales se refleja en algunas dimensiones como la intensidad de algunos usos decisivos y la experiencia en la utilización de estas herramientas. El análisis propuesto en este artículo ha sido posible gracias a la aplicación de un cuestionario, aunque la investigación completa se llevó a efecto complementando el trabajo de campo con otras técnicas de recogida de datos. Como se dijo al principio, el cuestionario permite obtener información sobre un número amplio de unidades muestrales de análisis en un espacio temporal concreto con la intención de conseguir de forma sistemática un cuerpo de datos cuantificable, respecto de un número determinado de dimensiones o variables que se examinan para establecer patrones de asociación entre ellas. Los estudios que se centran en las tecnologías dentro de las Administraciones locales también han asumido esta técnica con sus ventajas y problemas, de manera que en la siguiente sección se establecen unas breves reflexiones sobre las posibilidades de uso del cuestionario en el análisis de la e-Administración local, teniendo en cuenta los resultados de este trabajo y los estudios antes brevemente presentados.

\section{REFLEXIONES SOBRE EL CUESTIONARIO Y LA $e$-ADMINISTRACIÓN LOCAL}

Como ya se ha planteado, los cuestionarios se han utilizado de manera tradicional en el análisis de las organizaciones, en general, y las Administraciones Públicas, en particular. La aplicación concreta de los cuestionarios a las Administraciones locales se puede hacer en diferentes contextos institucionales, teniendo en cuenta distintos objetivos de investigación y tratando de evaluar variables y dimensiones variadas. En todo caso, los cuestionarios cuentan con diferentes ventajas y problemas genéricos, como técnica de recogida de datos, y particulares, en su aplicación específica al análisis de las Administraciones locales, que se han de sopesar a la hora de decidir la adopción de este tipo de herramieta en un diseño de investigación. Si se plantea como objeto de estudio la $e$-Administración local son precisas además algunas reflexiones específicas sobre la aplicación de esta técnica de recogida de datos. En esta sección se abordan estos temas de forma muy resumida.

Tradicionalmente, se han planteado una serie de oportunidades e inconvenientes vinculados a los cuestionarios en investigaciones organizativas, aunque aquí se exponen teniendo en cuenta su aplicación en diseños orientados de manera específica al estudio de las Administraciones locales. Por una parte, se suelen manejar una serie de argumentos para justificar su uso, entre los que se pueden citar los siguientes: 
(a) La posibilidad de obtener datos agregados para realizar análisis de carácter estadístico. La existencia de un elevado número de unidades de análisis dentro del nivel administrativo local facilita el uso del cuestionario, ya que proporciona la posibilidad de contar con un elevado N, incluso si la investigación se limita a un solo país o una sola región, lo que enlaza bien con el carácter netamente cuantitativista de muchas de estas aproximaciones y la creación de modelos cuasi-experimentales como metodología para la contrastación de hipótesis.

(b) El acceso a un número elevado de Administraciones locales resulta a veces inviable mediante otro tipo de técnicas de recogida de datos. Las entrevistas en profundidad o la observación participante a veces están limitadas por el número de organizaciones que se pueden visitar físicamente durante una investigación, por ello, el cuestionario facilita un estudio agregado de un conjunto más elevado de Administraciones locales, más si cabe en aquellos casos en los que se cubre un ámbito geográfico amplio que pone mayores dificultades para el acceso a información dentro de este nivel administrativo.

(c) Las Administraciones locales suelen ofrecer una apertura apreciable dado su carácter público. A diferencia de muchos sectores de la empresa privada, las Administraciones locales son organismos públicos cuyos datos suelen estar disponibles en directorios oficiales, registros $u$ otros medios de acceso público, de manera que la información sobre su localización, estructura, puestos directivos, personal a su servicio, recursos generales, etc., en principio, debería estar disponible para cualquier ciudadano. No obstante, la calidad de las respuestas y el éxito del cuestionario dependen de la realidad institucional y de las prácticas del sistema político, la existencia de respaldo institucional para la investigación, el perfil de las personas a las que se envíe u otro tipo de causas vinculadas a la oportunidad o el momento.

Por otra parte, el cuestionario no está exento de una serie de problemas en su aplicación dentro de estudios sobre Administraciones locales, algunos de los cuales se pueden resumir en los siguientes aspectos:

(a) El hecho de disponer de muestras amplias no garantiza que las inferencias estadísticas sean siempre robustas. La aplicación de los cuestionarios en las Administraciones locales se enfrenta a la doble problemática de realizar un diseño muestral adecuado y contar con un número de respuestas suficiente para realizar inferencias causales relevantes sin contener sesgos. El diseño muestral de las Administraciones locales objeto de estudio tiene que considerar la existencia de características específicas 
que pueden introducir sesgos en el análisis, lo que a veces no es fácil de controlar cuando se trata de estudios amplios que abordan países o regiones diferentes con realidades institucionales muy concretas que a veces quedan fuera del alcance de la investigadora. Por su parte, el problema de la representatividad de las respuestas obtenidas sugiere cuidar el alcance de las generalizaciones, ya que pueden proceder de inferencias estadísticas espurias si existen características entre los respondentes que introducen sesgos no tenidos en cuenta.

(b) El tamaño de los cuestionarios limita el número de variables del análisis y, por tanto, el nivel de detalle en una investigación organizativa. La posibilidad de contar con un elevado número de casos a la hora de estudiar Administraciones locales suele implicar la renuncia a una mayor profundidad en el análisis organizativo, es decir, esta técnica de recogida de datos sólo se puede emplear para arrojar luz acerca de determinados fenómenos muy específicos de las Administraciones locales, reduciendo la posibilidad de conclusiones más detalladas o asociaciones más específicas entre un mayor número de variables, ya que no se tiene una perspectiva de conjunto sobre la realidad institucional concreta en la que se insertan los fenómenos objeto de análisis.

(c) Por otra parte, los cuestionarios se enfrentan al inframunicipalismo propio de muchos contextos institucionales como el español. El hecho de contar con Administraciones locales de pequeño tamaño supone que su volumen institucional y humano suele ser muy limitado, por ello hay que ser consciente de esta realidad para conocer los límites de este tipo de herramienta. De hecho, muchas de las investigaciones suelen limitar el universo de Administraciones locales en función del tamaño de las ciudades donde se ubican (más de 2000, 10000, 20000 ó 50000 habitantes, o bien un número determinado entre los municipios de mayor tamaño, etc.) con el objetivo de orientarse a organizaciones con un mínimo desarrollo institucional y humano.

Teniendo presentes esos aspectos generales sobre las ventajas y problemas de los cuestionarios para el estudio de las Administraciones locales, lo interesante aquí consiste en mencionar al menos una serie de reflexiones específicas sobre su uso en el análisis de la $e$-Administración local. A la luz de los estudios antes mencionados, de la propia experiencia en este tipo de investigaciones y los resultados del trabajo presentado en este artículo se pueden apuntar una serie de conclusiones sobre los cuestionarios en el análisis de las tecnologías dentro del sector público local.

En primer lugar, la relativa facilidad para aplicar cuestionarios está provocando la proliferación de estudios aislados procedentes de organismos 
públicos, consultoras o centros de investigación, que cuentan con motivaciones y objetivos muy diferentes (lo cual es lógico y loable), aunque se tienden a asimilar sus resultados sin una reflexión sobre las diferencias de diseño, que no se suelen explicitar. Quizá sería conveniente plantear de manera más nítida esas motivaciones y objetivos, con lo que los lectores tendrían más fácil seleccionar los textos según sus propios intereses y necesidades.

Unido a lo anterior, la falta de diálogo entre las investigaciones académicas sobre $e$-Administración local limita las posibilidades de los cuestionarios como herramienta de investigación de esta realidad. La falta de fluidez en la comunicación de los trabajos académicos conlleva serios problemas para establecer un carácter longitudinal a los hallazgos, aprovechar las evidencias empíricas anteriores o establecer un programa amplio de conclusiones más generalizable a diferentes contextos institucionales. En este sentido, la aparición de revistas y congresos específicamente dedicados a este tema colaborarán en la mejora de esta situación.

Por otra parte, los cuestionarios suelen encerrar problemas de consistencia interna relacionados con su propio diseño o su relación con la teoría que soporta toda la investigación. Los cuestionarios contienen a veces un diseño de las preguntas inadecuado que resta consistencia a la herramienta, además, no siempre están presentes los objetivos y las preguntas de investigación a la hora de configurar y aplicar el cuestionario, de manera que se pueda establecer una conexión lógica con la teoría o teorías subyacentes. En este sentido, hay que tener en cuenta el carácter híbrido de los conocimientos necesarios para el diseño de un cuestionario sobre $e$ Administración local. Este objeto de estudio implica la necesidad de disponer de suficientes conocimientos sobre el contexto tecnológico, pero también teniendo en cuenta que se están analizando organizaciones que tienen una naturaleza político-administrativa que hay que dominar con cierta profundidad, sobre todo, para evitar las limitaciones de aquellos estudios que eluden esta peculiaridad trasladando de forma mimética experiencias de análisis procedentes de la empresa privada o que inciden de manera exclusiva en componentes vinculados con la tecnología.

Finalmente, aunque no se pretende abundar en problemas sobre nominalismos, parece que todavía no está del todo claro lo que se está estudiando cuando se habla de $e$-Gobierno, $e$-Democracia, $e$-Gobernanza o $e$-Administración local. Más allá de las interpretaciones particulares que se puedan hacer sobre el alcance de estos términos, la cuestión es que en muchos casos no se es siquiera consciente de este problema, de manera que esa imprecisión conceptual puede impedir la construcción de una 
comunidad de realidades y significados compartidos entre todos aquellos que participan en la esfera político-administrativa local. Y este hecho resulta decisivo para mejorar la calidad de cualquier investigación sobre $e$-Administración local, especialmente, en tanto se precisen las respuestas a un cuestionario como medio para adquirir conocimiento sobre ese objeto de estudio. Pero este debate queda fuera de los límites de este artículo.

\section{CONCLUSIÓN}

Aquí se ha mostrado que el análisis de las tecnologías en las Administraciones locales tiene en los cuestionarios una herramienta con tradición y que todavía hoy se usa con cierto éxito. Las investigaciones que utilizan cuestionarios han crecido de manera importante desde los años 1960 como consecuencia de la ola cuantitativista que se extendió desde las universidades estadounidenses dentro de los estudios sociales, políticos y administrativos. Los cuestionarios se han aplicado de manera extensiva en los estudios organizativos y, en concreto, en el análisis de las Administraciones Públicas, de forma que el tema de las tecnologías en el sector público local tampoco ha sido ajeno a esta tendencia, como se ha puesto de relieve a través de un repaso realizado a algunas de las investigaciones más relevantes publicadas en las tres últimas décadas. Teniendo como punto de referencia esas aportaciones, se ha presentado una investigación sobre la e-Administración local en los municipios de la Comunidad de Madrid, en concreto, algunos de los resultados derivados de la aplicación de un cuestionario a los gestores de las TIC en las organizaciones objeto de estudio sobre varias dimensiones de interés, lo que ha permitido plantear conclusiones de carácter preliminar y descriptivo. Finalmente, el artículo ha puesto de relieve determinados problemas y ventajas del uso de los cuestionarios en estudios centrados en las Administraciones locales, ampliando esta cuestión con unas reflexiones sobre la $e$-Administración en esa esfera político-administrativa, tema de investigación que tiene en el cuestionario un aliado que se puede y debe utilizar cuando el objeto de estudio así lo precise y, siempre que sea posible, conjuntamente con otras técnicas de recogida de datos.

\section{BIBLIOGRAFÍA}

Anduiza, Eva, Ismael Crespo, y Mónica MÉndez, 1999: Metodología de la Ciencia Política. Madrid: Centro de Investigaciones Sociológicas. 
Arenilla, Manuel, 2003: El Estado y la Administración Pública en la Sociedad de la Información. Bole.tic 25:14-25.

Azofra, M. José, 1999: Cuestionarios. Madrid: Centro de Investigaciones Sociológicas.

Bellamy, Christine, y John A. TAYLoR; 1998: Governing in the Information Age. Buckingham: Open University Press.

Bryman, Alan, 1989: Research Methods and Organizational Studies. London: Unwin Hyman.

CASTElls, Manuel, y Esteve Ollé, 2004: El Model Barcelona II: L'Ajuntament de Barcelona a la Societat Xarxa. Barcelona: Universitat Oberta de Catalunya.

Criado, J. Ignacio, 2004a: Construyendo la e-Administración Local. Madrid: EuroGestión Pública.

Criado, J. Ignacio: 2004b: Entre Sueños Utópicos y Visiones Pesimistas. Un Análisis de la Administración Electrónica Local en España. Gestión y Política Pública 13 (2):469-524.

CRiado, J. Ignacio: 2003: ¿Retórica o Realidad? La Promoción de la eAdministración en España. Una Aproximación a la Situación en la Administración General del Estado. Prospectiva 25:11-22.

Criado, J. Ignacio, y M. Carmen Ramilo, 2003a: e-Government in Practice. An Analysis of Web Site Orientation to the Citizens in Spanish Municipalities. International Journal of Public Sector Management 16 (3):191-218.

Criado, J. Ignacio, y M. Carmen RAmilo, 2003b: Gobierno y Administración Pública en la Sociedad de la Información. Análisis Local 51 (VI):59-74.

Criado, J. Ignacio, y M. Carmen Ramilo, 2001: Definiendo la e-Administración. Las Páginas Web de las Administraciones Públicas. Gestión y Análisis de Políticas Públicas 22:123-138.

Danziger, James N., Kenneth L. Kraemer, Debora E. Dunkle, y John L. KING, 1993: Enhancing the Quality of Computing Service: Technology, Structure and People. Public Administration Review 53 (2):161169. 
Danziger, James N., William H. Dutton, Rob Kling, y Kenneth L. Kraemer, eds., 1982: Computers and Politics. High Techology in American Local Governments. Ed. New York: Columbia University Press.

Danziger, James N., y Kenneth L. Kraemer, 1986: People and Computers. The Impacts of Computing on End Users in Organizations. New York: Columbia University Press.

DANZIGER, James N., y William H. DutTon, 1977: Computers as an Innovation in American Local Governments. Communications of the ACM 20 (12):945-956.

Dunleavy, Patrick, y Hellen Margetts, 2002: Government on the Web 2002. London: National Audit Office.

Dutton, William H., y Kenneth L. Kraemer, 1978: Management Utilization of Computers in American Local Governments. Communications of the ACM 21 (3):206-218.

FundaCión RETEVISIÓn, 2004: España 2004. Informe Anual sobre el Desarrollo de la Sociedad de la Información en España. Madrid: Fundación Retevisión.

HEEKs, Richard, ed., 1999: Reinventing Government in the Information Age. International Practice in IT-Enable Public Sector Reform. London: Routledge.

Ho, Alfred T., 2002: Reinventing Local Governments and the e-Government Initiative. Public Administration Review 62 (4):434-444.

ICMA. 1993: Information Technology Survey. Washington D. C. International City/County Management Association.

ICMA. 1997: Information Technology Survey. Washington D. C. International City/County Management Association.

KING, John L., y Kenneth L. Kraemer, 1991: Patterns of Success in Municipal Information Systems: Lessons from US Experience. Informatization and the Public Sector 1 (1):21-39.

KRAEMER, Kenneth L., 1971: A Model for Urban Information Systems. En Information Technology in a Democracy, ed. A. F. Westin. Cambridge: Harvard University Press. 
La Porte, Todd M. , Chris C. Demchak, y Martin De Jong, 2002: Democracy And Bureaucracy In The Age Of The Web: Empirical Findings and Theoretical Speculations. Administration \& Society 34 (4):411446.

MARGETTS, Hellen, 1999: Information Technology in Government: Britain and America. London: Routledge.

Margetts, Hellen, y Patrick Dunleavy, 2002: Better Public Services Through e-Government. London: National Audit Office.

MAP., 2002: La Informática en las Administraciones Públicas. Informe IRIA-2002. Madrid: Ministerio de Administraciones Públicas - Boletín Oficial del Estado.

MAP., 1993: La Informática en las Administraciones Públicas. Informe IRIA-92. Madrid: Ministerio de Administraciones Públicas.

Moon, M. Jae, 2002: The Evolución of e-Government among Municipalities: Rhetoric or Reality? Public Administration Review 62 (4):424-433.

Musso, Juliet A., Christopher Weare, y Matthew L. Hale, 2000: Designing Web Technologies for Local Governance Reform: Good Management or Good Democracy? Political Communication 17 (1):1-19.

NorRIS, Donald F., 1999: Leading Edge Information Technologies and Their Adoption: Lessons from U.S. Cities. En Information Technology and Computer Applications in Public Administration: Issues and Trends, ed. G. D. Garson. Hershey: Publishing Idea Group.

Northrop, Alana, Kenneth L. Kraemer, Debora E. DunkLe, y John L. KING, 1990: Payoffs from Computerization: Lessons over Time. Public Administration Review 50 (5):505-514.

Ramió, Carles. 2002. El Papel de la e-Administración ante los Retos de las Administraciones Públicas de América Latina: e-Administración y Sector Público en el Siglo de la Globalización. Ponencia para el VII Congreso Internacional del CLAD, 8-11 de octubre, Lisboa.

Salvador, Miquel, 2004: Els Ajuntaments de Catalunya à Internet. Un Estudi Comparat de les Pàgines Web (2000-2003). Barcelona: Universitat Pompeu Fabra.

Scavo, Carmine, y Shi Yuhang, 1999: World Wide Web Design and Use in Public Management. En Information Technology and Computer 
Applications in Public Administration: Issues and Trends, ed. G. D. Garson. Heshey: Publishing Idea Group.

WINNER, L., 1977: Autonomous Technology. Cambridge: MIT Press. 


\section{Jurisprudencia}

